\title{
Observation of a short-lived pattern in the solar chromosphere
}

\author{
F. Wöger, S. Wedemeyer-Böhm, W. Schmidt, and O. von der Lühe
}

Kiepenheuer-Institut für Sonnenphysik, Schöneckstraße 6, 79104 Freiburg, Germany

e-mail: [woeger, wedemeyer, wolfgang, ovdluhe] @kis.uni-freiburg.de

Received 11 August 2006 / Accepted 13 September 2006

ABSTRACT

\begin{abstract}
Aims. In this work we investigate the dynamic behavior of inter-network regions of the solar chromosphere. Methods. We observed the chromosphere of the quiet Sun using a narrow-band Lyot filter centered at the Ca II $\mathrm{K}_{2 \mathrm{v}}$ emission peak with a bandpass of $0.3 \AA$. We achieved a spatial resolution of on average 0.7 at a cadence of $10 \mathrm{~s}$.

Results. In the inter-network we find a mesh-like pattern that features bright grains at the vertices. The pattern has a typical spatial scale of 1".95 and a mean evolution time scale of $53 \mathrm{~s}$ with a standard deviation of $10 \mathrm{~s}$. A comparison of our results with a recent threedimensional radiation hydrodynamical model implies that the observed pattern is of chromospheric origin. The measured time scales are not compatible with those of reversed granulation in the photosphere although the appearance is similar. A direct comparison between network and inter-network structure shows that their typical time scales differ by at least a factor of two.

Conclusions. The existence of a rapidly evolving small-scale pattern in the inter-network regions supports the picture of the lower chromosphere as a highly dynamical and intermittent phenomenon.
\end{abstract}

Key words. Sun: chromosphere

\section{Introduction}

Deducing the thermal structure of the solar chromosphere is complicated by the limited number of diagnostics and the difficulties in interpreting them. The core regions of the calcium resonance lines ( $\mathrm{Ca} \mathrm{II} \mathrm{H}$ and $\mathrm{K}$ ) that are commonly assumed to originate from the chromosphere (cf. Vernazza et al. 1981) are an example. Unfortunately the simplifying assumption of local thermodynamic equilibrium (LTE) is not valid for the emission reversal peaks and the cores of these lines. Rather, non-local effects must be taken into account for a realistic description of chromospheric plasma and radiation fields. This is not only a problem for the interpretation of observations of these spectral features, but also for the numerical modeling of the chromosphere. The recent three-dimensional radiation hydrodynamics model by Wedemeyer et al. (2004, hereafter W04) shows a mesh-like pattern at chromospheric heights in non-magnetic inter-network regions, consisting of cool regions and hot filaments that are a product of propagation and interaction of oblique shock waves and adiabatic expansion of post-shock regions. The authors argue that the amplitude of the temperature variations in the model chromosphere is somewhat uncertain due to the too simple radiative transfer (grey, LTE). But the pattern is nevertheless representative as it is a product of acoustic waves that are excited in the lower well-modeled layers. The spatial scales of the pattern are comparable to the underlying granulation, but the dynamical time scales are much shorter. Mapping the chromospheric small-scale structure of inter-network regions thus requires both high spatial and temporal resolution. This might be the reason why most earlier observations failed to detect this phenomenon (Table 1).

Here we present new observations taken with a Lyot filter of very narrow transmitted wavelength range and a spatial resolution high enough to resolve the chromospheric small-scale
Table 1. Recent observations of the Ca II resonance lines. The properties of the data show that the presented data set is unique regarding the combination of spectral and spatial resolution $(\Delta \lambda$ is $F W H M)$.

\begin{tabular}{lrrrr}
\hline \hline Author & $\begin{array}{r}\Delta \lambda \\
{[\AA]}\end{array}$ & $\begin{array}{r}\text { Scale } \\
{[\AA]}\end{array}$ & $\begin{array}{r}\Delta \mathrm{t} \\
{\left[{ }^{\prime \prime} / \mathrm{px}\right]}\end{array}$ & {$[\mathrm{s}]$} \\
\hline This work & 3933.5 & 0.30 & 0.1485 & 10 \\
Rutten et al. (2004) & 3968.8 & 1.35 & 0.071 & 30 \\
Tritschler \& Schmidt (2002) & 3933.2 & 0.60 & 0.350 & 6 \\
De Pontieu (2002) & 3933.0 & 3.00 & 0.083 & $\sim 20$ \\
Kneer \& von Uexküll (1993) & 3933.0 & 0.60 & 0.720 & 15 \\
\hline
\end{tabular}

pattern of inter-network regions. In Sect. 2 we describe the observations and data reduction, followed by results and discussion in Sects. 3 and 4, respectively.

\section{Observation and data reduction}

A high-resolution image sequence was obtained with the German Vacuum Tower Telescope (VTT) at the Observatorio del Teide on Tenerife, Spain, during UT 8:25-10:21 on April 18, 2005 . The telescope was pointed at a quiet area near the diskcenter. The field of view is $49^{\prime \prime} \times 49^{\prime \prime}$, showing the chromospheric network and inter-network. The data set consists of images observed in the G-band (4305 $\AA$ ) using an interference filter with a $F W H M$ of $10 \AA$, and, using Lyot filters, in Ca II K (3933 $\AA$ ) and H $\alpha(6563 \AA)$ with FWHMs of $0.3 \AA$ and $0.25 \AA$, taken simultaneously. In this paper we only consider the results obtained from the calcium filtergrams. A sample image together with close-ups of a network and an inter-network region is shown in Fig. 1. The filter curve of the $\mathrm{Ca}$ filter is displayed in Fig. 2 along with filter curves of other observations for comparison. The image sequence was taken at a cadence of $10 \mathrm{~s}$ and a pixel scale 
A
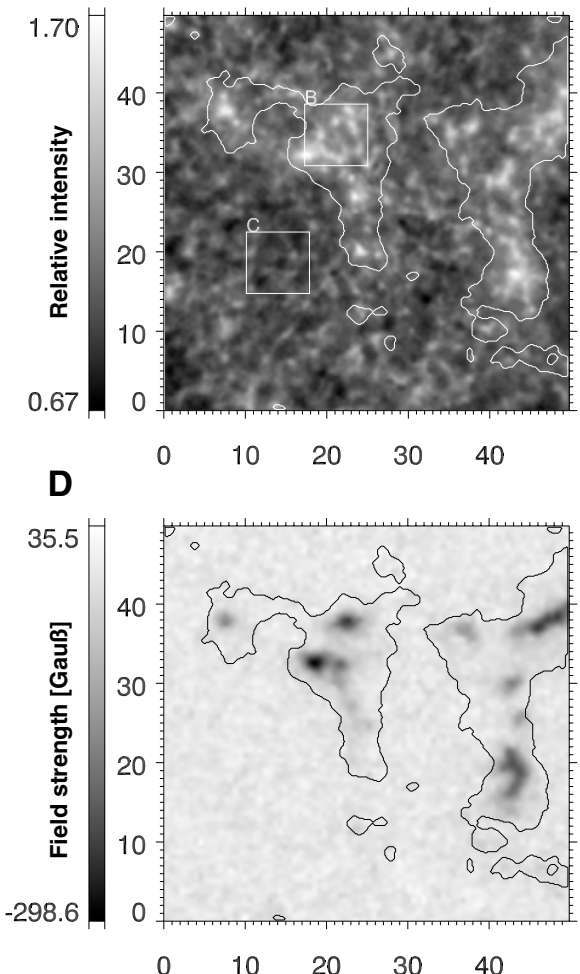

B

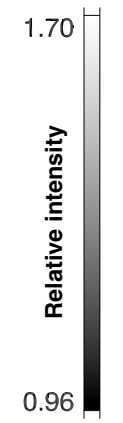

E

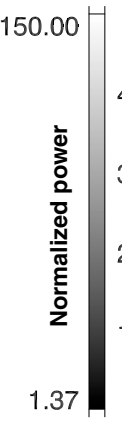

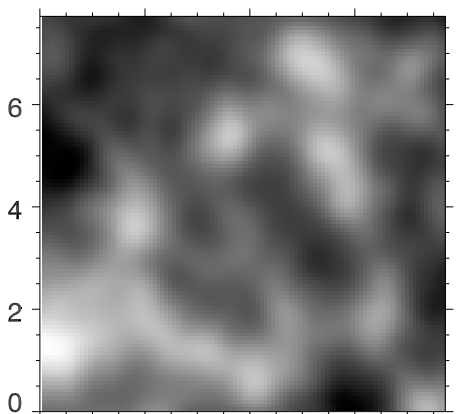

$\begin{array}{llll}0 & 2 & 4 & 6\end{array}$

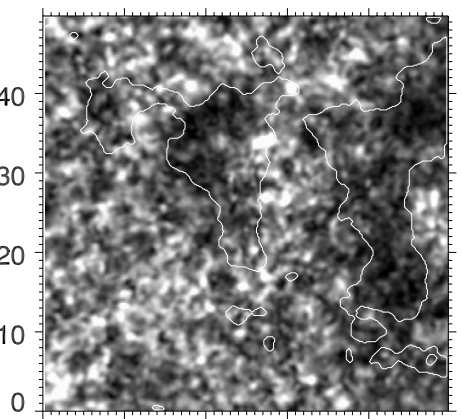

$0 \quad 10 \quad 20 \quad 30 \quad 40$

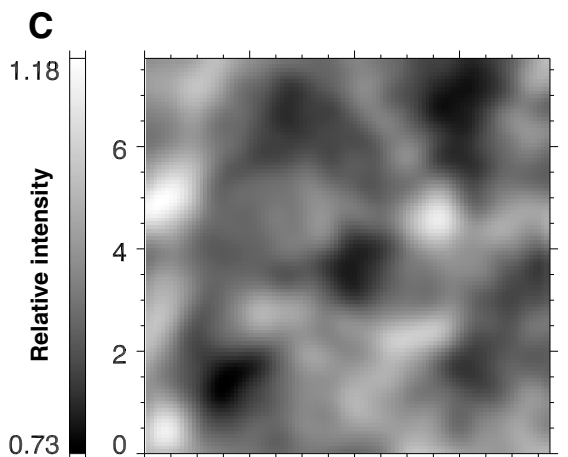

0.73
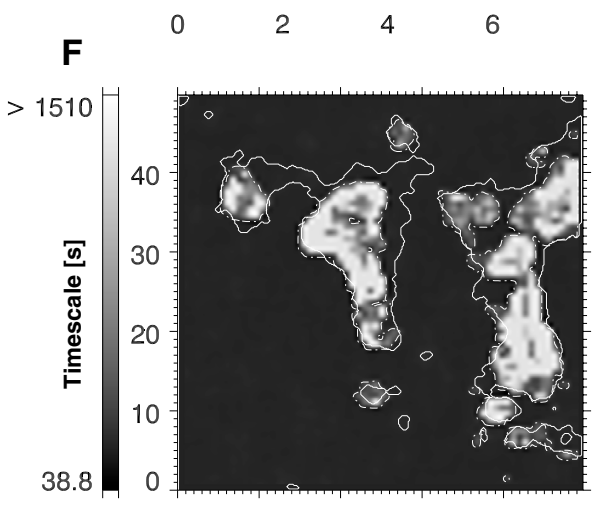

$\begin{array}{lllll}0 & 10 & 20 & 30 & 40\end{array}$

Fig. 1. (A) Calcium image with superimposed network mask M1 and close-up regions (squares), (B) the close-ups of the network region and (C) the inter-network region, (D) the MDI magnetogram, (E) a 2D power map of the temporal frequency band at $5.5 \mathrm{mHz}$ with a width of $0.9 \mathrm{mHz}$, and (F) the map of 1/e time scales, together with M1 (solid contour) and the new network mask M2 (dot-dashed contour). All axes are in arcseconds.

of $0.1485 / \mathrm{px}$. There was a need for telescope adjustments at UT 9:00, separating the sequence into two parts with a gap of $2 \mathrm{~min}$. The transmission of the Lyot filter is rather poor, thus the light level of the Ca II K line core - being by itself less than 5\% of the continuum intensity - was reduced even further, leading to an exposure time of $2 \mathrm{~s}$.

All images were corrected for hot pixels and calibrated using the standard procedure of flat-fielding. Additionally, the sequence was corrected for the change in mean intensity in each image due to the increasing solar elevation with a fitted sine curve and scaled to the mean intensity of the time series. Finally, the noise in each image was reduced using a Wiener Filter. From the cutoff of the azimuthally integrated spatial power spectrum we estimate a spatial resolution of on average 0.7 or better, which was achieved by the fair to good seeing $\left(r_{0} \approx 13 \mathrm{~cm}\right)$ in combination with the Kiepenheuer Adaptive Optics System (KAOS).

\section{Analysis and results}

In our analysis we focus on the second half of the image series with a length of $75 \mathrm{~min}$. Network regions embody chains of persistent bright points (Fig. 1B). Inter-network regions show a highly dynamic behavior with small-scale, cell-like structures (Fig. 1C) and small brightenings at the vertices that resemble bright points or grains (see, e.g., Lites et al. 1999). We computed the spatial power spectrum of the inter-network pattern. To enhance the small-scale nature of the pattern of interest, an unsharp-masked image was subtracted from the data. The twodimensional power spectrum of the resulting image was then azimuthally integrated. The one-dimensional power spectrum is shown in Fig. 3. The barycenter of power is at spatial frequency $f_{h}=0.512 \operatorname{arcs}^{-1}$ corresponding to $1^{\prime \prime} 95$. The bright grains typically have a size of $1-2^{\prime \prime}$ (see Fig. 1C).

To demonstrate the dynamical behavior in further detail, we calculated the two-dimensional autocorrelation function for subfields of 1 '. $485 \times 1$ '. 485 , which overlap by half of their field size in both directions. The evolution time scale $\zeta_{\mathrm{pe}}$ is defined as the time at which the autocorrelation has dropped to a value of 1/e. Finding the evolution time scale for each subfield separately results in an array of $66 \times 66$ values over the field of view. The map in Fig. 1F shows the results for the individual subfields. Figure 4 shows a plot of time scale versus mean intensity for all subfields. To pursue a more detailed analysis of their different behavior, criteria that distinguish network and inter-network regions need to be established.

As a first step, the lower mean brightness of the internetwork pattern was used to create a mask of network and internetwork regions by thresholding the smoothed temporal mean of the intensity (cf. Rutten et al. 2004). We found such a threshold at $I=1.017$ by fitting the sum of two Gaussians to the intensity histogram of the mean image. In Fig. 4, the threshold is indicated and divides the plot into two regions, the left containing dark subfields, the right containing the bright subfields. The application of the threshold in combination with a morphological operation to remove isolated pixels resulted in mask M1, which is indicated in Figs. 1A, D, E and F. Interestingly, the time scale distribution of subfields in Fig. 4 shows a gap between $100 \mathrm{~s}$ and $200 \mathrm{~s}$ that separates long and short time scales. We therefore chose a threshold of $150 \mathrm{~s}$ to discriminate between the fast and the slow evolving regimes (see Fig. 4). Together with the intensity threshold, the new time scale criterion divides the plot into quadrants. Applying the time scale threshold to the image sequence results in a new mask M2, which is indicated in Fig. 1F. 


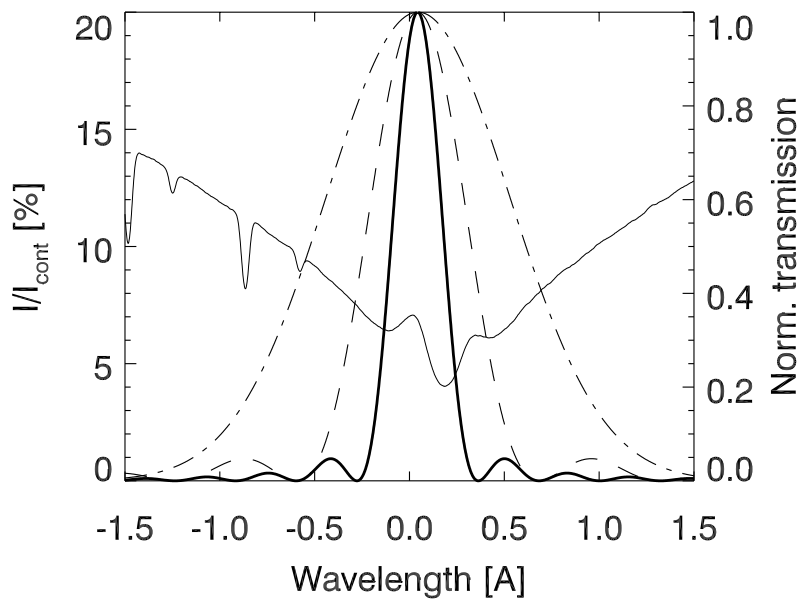

Fig. 2. The calcium line around $3933.5 \AA$ (Neckel 1999) (thin solid) plotted together with theoretical Lyot filter transmission curves with $0.3 \AA$ (thick solid), $0.6 \AA$ (dashed) and a Gaussian with $1.350 \AA$ (dotdashed) $F W H M$ (see Table 1).

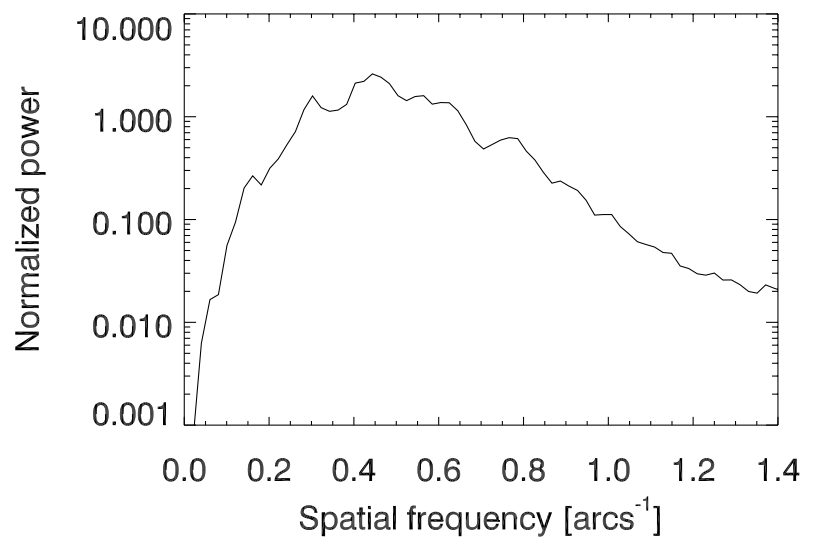

Fig. 3. One-dimensional power spectrum of the inter-network pattern.

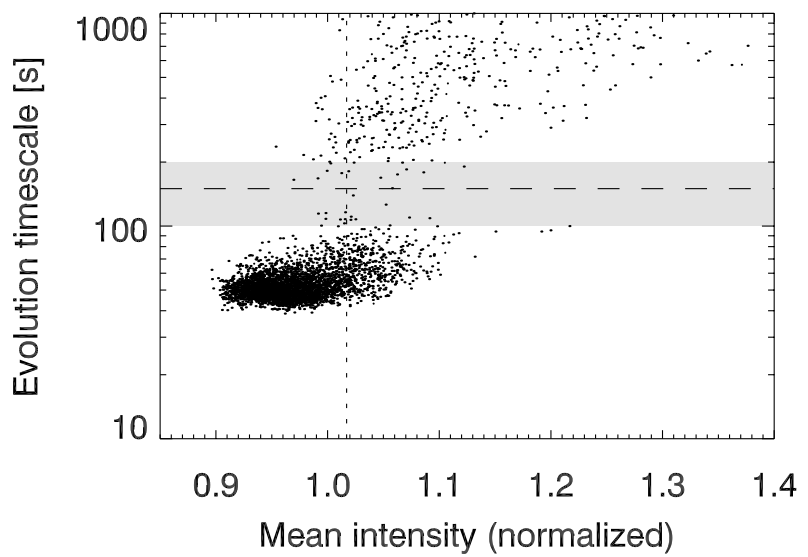

Fig. 4. Time scale versus intensity. The gap between 100 and $200 \mathrm{~s}$ indicated with a shaded horizontal band shows the different behavior of network and inter-network structures. The vertical line (dotted) indicates the intensity threshold used for the creation of M1, the horizontal line (dashed) the temporal threshold for M2.

Mask M2 is almost completely contained within mask M1. The pixels that are attributed to the network in mask M1, but not in $\mathrm{M} 2$, belong to subfields that contribute to the lower right quadrant of Fig. 4. This quadrant contains subfields that are bright on average and exhibit short time scales. Apparently, there are many

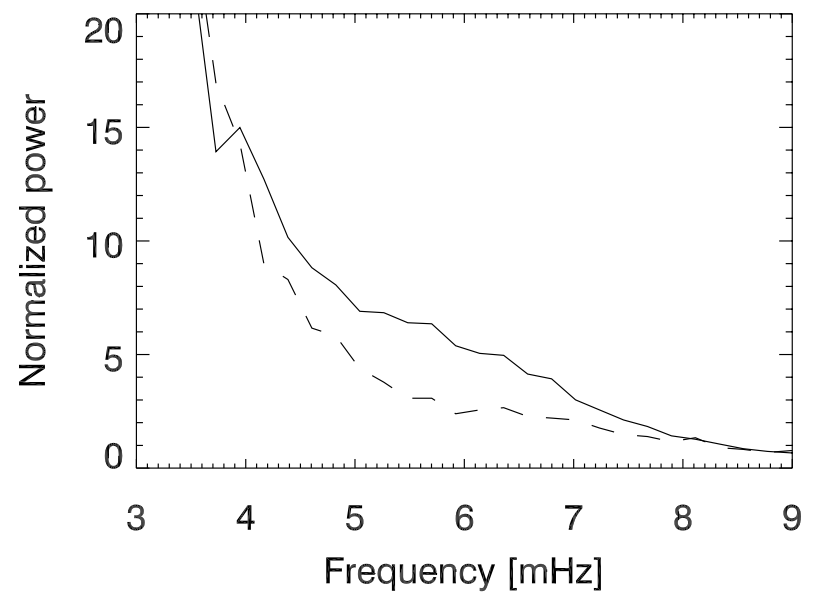

Fig. 5. Temporal power for network (dashed) and inter-network (solid) regions.

structures which belong to the inter-network even though they are brighter than average. That includes subfields with bright $\mathrm{Ca}$ II $\mathrm{K}$ grains, implying a connection between grains and internetwork pattern. The majority of fast evolving subfields, however, are darker than average and clearly belong to inter-network regions (lower left quadrant). The barycenter of these subfields (with time scales below $150 \mathrm{~s}$ ) lies at $\overline{\zeta_{\text {pe }}}=53 \mathrm{~s}$ and has a normalized intensity of 0.953 . The standard deviation in time scale is $10 \mathrm{~s}$, and the minimum value is $\zeta_{\text {pe,min }}=39$ s. In contrast, network structures show long time scales, some of which were longer than the duration of our data sequence, and are on average brighter than inter-network structures. Therefore, they are found mostly in the upper right quadrant of Fig. 4. As expected, these persistent patches of enhanced intensity match magnetic structures shown in the MDI magnetogram (Fig. 1D), identifying them as part of the network. There are only a few dark and slowly changing structures that appear in the upper left quadrant.

We computed temporal power spectra for each pixel and averaged them over network and inter-network pixels according to mask M2. The result in Fig. 5 shows a clearly enhanced signal in the frequency band around $5.5 \mathrm{mHz}$ within inter-network regions. This can also be seen in the power map of Fig. 1E, where the band around $5.5 \mathrm{mHz}$ with a band pass of $0.9 \mathrm{mHz}$ is displayed. The network mask is again outlined by contour lines. The pixels of decreased power are almost completely contained within M2.

\section{Discussion and conclusion}

We provide a new method to discriminate network and internetwork structures in filtergrams that show chromospheric structure when the data are not accompanied by information about the underlying magnetic field. Figure 4 demonstrates that the pattern evolution time scale provides an excellent criterion to distinguish between network and inter-network regions: all inter-network points are grouped in the time interval below $100 \mathrm{~s}$, whereas the network has time scales above $200 \mathrm{~s}$. Temporal thresholding delivers an accurate mask, M2, for network pixels.

Structures in the inter-network similar to those found in our observations have already been detected in various other works. Leenaarts \& Wedemeyer-Böhm (2005, hereafter LW05) investigated a similar pattern at higher spatial resolution - but lower spectral resolution - and identified it with reversed granulation. This result is also supported by the much longer time scale of 
2 min they determined for the pattern. In Fig. 1 of Kneer \& von Uexküll (1993), the pattern can be surmised. Tritschler \& Schmidt (2002) present observational data with time scales of inter-network structures close to the ones obtained in this work. Their spatial resolution did not suffice to resolve the small-scale pattern, and they used a broader bandwidth of $0.6 \AA$. This work is the first one to observe the small-scale pattern with a mean evolution time scale much shorter than $100 \mathrm{~s}$.

To verify the chromospheric origin of the detected internetwork pattern, we compare its spatial and evolution time scale to the recent 3D model by W04. The temperature amplitudes of the model chromosphere might be somewhat uncertain due to the simplified treatment (grey, LTE), whereas the excitation of the waves occurs in realistically modeled lower layers, making the dynamics and related time scales more reliable. A direct comparison with synthetic intensity maps for $\mathrm{Ca}$ II $\mathrm{K}_{2 \mathrm{v}}$ is not possible yet as the model chromosphere is dominated by strong shocks that cause numerical problems. We can, however, perform a qualitative comparison with horizontal slices of gas temperature from the model chromosphere. The cuts exhibit a pattern of granular size at a geometrical height around $1000 \mathrm{~km}$ above $\tau=1$, which rapidly evolves with time scales of around $\zeta_{\mathrm{pe}}=20-30 \mathrm{~s}$. The pattern is quite similar to the one we observe, but the evolution time scale is still a factor of two shorter than the observational value. This can be explained as follows: first of all, the correlation between gas temperature at $z=1000 \mathrm{~km}$ and intensity in the Ca II K $\mathrm{K}_{2 \mathrm{v}}$ spectral feature might be poor as the assumption of LTE is not valid in this strongly scattering line. In LTE local changes in temperature are instantaneously translated into changes, of the source function and thus intensity changes although the intensity response is non-linear in amplitude. In contrast to the temperature slices at a well-defined geometrical height, the emergent intensity (at a given wavelength) is a non-local quantity that has contributions from an extended height range. For the line core, the main contribution of intensity originates from chromospheric heights around $1000-1500 \mathrm{~km}$ above $\tau=1$ (cf. Vernazza et al. 1981), but already at $0.15 \AA$ off-core, considerable contributions to the filtergram intensity from lower geometrical heights have to be expected (Rammacher, priv. comm.). The emergent intensity is thus integrated along the line of sight over many layers that individually would show different pattern evolution times (decreasing with height, see Wedemeyer 2003). The integrated pattern is consequently smoothed so that the relative intensity variations become smaller and result in longer evolution time scales. This effect is even enhanced due to the fact that the observed intensity refers to corrugated surfaces of optical depth instead of plane horizontal cuts as used for the model. The intensity pattern will be smoothed even more compared to the fine structure in the temperature cuts. W04 calculate a time scale of $\zeta_{\mathrm{pe}, z}>120 \mathrm{~s}$ for changes in gas temperature in a horizontal plane cut at the bottom of the photosphere, whereas the grey emergent intensity (under an assumption of LTE, which reflects conditions at the bottom of the photosphere), yields an increase of the typical time scale $\zeta_{\mathrm{pe}, \tau}=200 \mathrm{~s}$. This is due to the fact that variations tend to be smaller on surfaces of optical depth because they are shaped by inhomogeneities themselves. In addition, seeing and instrumental effects lead to a spatial smearing of the intensity signal and thus to longer time scales. We found that smoothing the temperature cuts to the spatial resolution encountered in our data increases the time scales by about $25 \%$. The simulations of W04 do not include magnetic fields. The existence of internetwork fields (with intrinsic field strengths varying from $1 \mathrm{kG}$ down to $\lesssim 100 \mathrm{G}$ ) is undisputed as they have been recently measured with high polarimetric precision (see Socas-Navarro et al. 2004; Lites \& Socas-Navarro 2004, and references therein). The contribution of possibly unresolved magnetic elements in internetwork regions tends to lengthen the effective evolution time scale. A preliminary analysis of temperature cuts from the 3D magnetohydrodynamics model by Schaffenberger et al. (2005) results in a slightly higher value of $\zeta_{\mathrm{pe}, \mathrm{MHD}}=27 \mathrm{~s}$ at the same height $(z=1000 \mathrm{~km})$ compared to $24 \mathrm{~s}$ in the purely hydrodynamical model by W04. Consideration of all these effects leads to a reasonable agreement between observation and numerical model. An interpretation of the pattern as reversed granulation is inappropriate as the corresponding time scale is much longer than the $53 \mathrm{~s}$ found here. LW05 state $120 \mathrm{~s}$ and $90 \mathrm{~s}$ for observed and synthetic $\mathrm{Ca}$ II H line wing intensities, respectively, which clearly originate from the mid-photosphere (LW05, Wedemeyer 2003). These values are in line with the time scale of the order of $90 \mathrm{~s}$ for the reversed granulation pattern observed in the Fe I line core at $709.0 \mathrm{~nm}$ by Janssen \& Cauzzi (2006). We conclude that the small-scale inter-network pattern reported here evolves too fast to be due to reversed granulation and is thus of chromospheric origin. The pattern is very likely the intensity signature of the mesh-like, fast evolving pattern found in recent 3D hydrodynamical models of the solar chromosphere. It is presumably a product of the propagation and interaction of shock waves (W04) that are excited below the observed layer. The same is true for the Ca II grains (see also Carlsson \& Stein 1997) that we consider an integral part of the pattern. Collision of two neighboring wave fronts results in compression and heating of the gas at the contact region that shows up as a band of enhanced intensity, making up the "strings" of the mesh-like pattern. At a mesh vertex, however, more than two wave fronts meet, resulting in stronger compression and thus in an intensity higher than the remaining pattern. Due to the limitations of earlier observations (see Table 1) only the brightest components have been detected as grains, whereas the pattern itself remained undetected. A more detailed study including the simultaneous G-band data is in preparation.

Acknowledgements. We thank A. Tritschler and R. Hammer for helpful discussions. K. Janssen kindly provided observational data for comparison. F. Wöger acknowledges support through the Landesgraduierten Förderungsgesetz des Landes Baden-Württemberg. S. Wedemeyer-Böhm was supported by the Deutsche Forschungsgemeinschaft (DFG), project Ste 615/5.

\section{References}

Carlsson, M., \& Stein, R. F. 1997, ApJ, 481, 500 De Pontieu, B. 2002, ApJ, 569, 474

Janssen, K., \& Cauzzi, G. 2006, A\&A, 450, 365 Kneer, F., \& von Uexküll, M. 1993, A\&A, 274, 584

Leenaarts, J., \& Wedemeyer-Böhm, S. 2005, A\&A, 431, 687 (LW05)

Lites, B. W., \& Socas-Navarro, H. 2004, ApJ, 613, 600

Lites, B. W., Rutten, R. J., \& Berger, T. E. 1999, ApJ, 517, 1013

Neckel, H. 1999, Sol. Phys., 184, 421

Rutten, R. J., de Wijn, A. G., \& Sütterlin, P. 2004, A\&A, 416, 333

Schaffenberger, W., Wedemeyer-Böhm, S., Steiner, O., \& Freytag, B. 2005, in ESA SP-596, ed. D. E. Innes, A. Lagg, \& S. A. Solanki

Socas-Navarro, H., Martínez Pillet, V., \& Lites, B. W. 2004, ApJ, 611, 1139

Tritschler, A., \& Schmidt, W. 2002, ESA SP-506, 785

Vernazza, J. E., Avrett, E. H., \& Loeser, R. 1981, ApJS, 45, 635

Wedemeyer, S. 2003, Ph.D. Thesis, Univ. Kiel,

http://e-diss.uni-kiel.de/diss_764/

Wedemeyer, S., Freytag, B., Steffen, M., Ludwig, H.-G., \& Holweger, H. 2004, A\&A, 414, 1121 (W04) 